\title{
Hyperelliptic integrals and generalized arithmetic-geometric mean
}

\author{
Jeroen Spandaw • Duco van Straten
}

Received: 31 March 2011 / Accepted: 17 October 2011 / Published online: 1 March 2012

(C) The Author(s) 2012. This article is published with open access at Springerlink.com

\begin{abstract}
We show how certain determinants of hyperelliptic periods can be computed using a generalized arithmetic-geometric mean iteration, whose initialisation parameters depend only on the position of the ramification points. Special attention is paid to the explicit form of this dependence and the signs occurring in the real domain.
\end{abstract}

Keywords Arithmetic-geometric means · Hyperelliptic integrals · Theta functions

Mathematics Subject Classification (2000) Primary 14K25 · Secondary 11B83 • $11 \mathrm{~F} 27 \cdot 14 \mathrm{H} 40$

\section{Introduction}

The study of hyperelliptic integrals

$$
\int R(x, \sqrt{f(x)}) \mathrm{d} x
$$

has a long and rich history and played a pivotal role in the development of the theory of Riemann surfaces and the algebraic geometry of curves. In this paper we consider

Part of the work was done with the support of the SFB/TR 45 during a stay of the first author at the Johannes Gutenberg University in Mainz.

J. Spandaw $(\bowtie)$

Delft University of Technology, Mekelweg 4, 2628 CD Delft, The Netherlands

e-mail: j.g.spandaw@tudelft.nl

D. van Straten

Institut für Mathematik, Johannes Gutenberg University, 55099 Mainz, Germany

e-mail: straten@mathematik.uni-mainz.de 
the classical topic of real hyperelliptic periods such as

$$
I_{j}\left(a_{1}, \ldots, a_{d}\right):=\int_{a_{j}}^{a_{j+1}} \frac{\mathrm{d} x}{\sqrt{\left|\left(x-a_{1}\right) \cdots\left(x-a_{d}\right)\right|}},
$$

where $a_{1}, \ldots, a_{d}$ are pairwise distinct real numbers. We will always assume that the branch points $a_{i}$ are ordered: $a_{1}<\cdots<a_{d}$. For $d=2$, these integrals are elementary:

$$
\int_{a}^{b} \frac{\mathrm{d} x}{\sqrt{(x-a)(b-x)}}=\pi
$$

For $d=3$ and $d=4$ they were studied by Lagrange in 1784 (see [15, p. 272]) and later by Gauss (see [11, pp. 352-353]), who related them to the arithmetic-geometric mean (agm). For a wonderful description of Gauss' theory, we refer to [5]. Recall that the agm of two positive real numbers $u$ and $v$ is defined using the sequences $\left(u_{n}\right)$ and $\left(v_{n}\right)$ defined recursively by $u_{n+1}:=\frac{1}{2}\left(u_{n}+v_{n}\right)$ and $v_{n+1}:=\sqrt{u_{n} v_{n}}$ starting with $u_{0}:=u$ and $v_{0}:=v$. It is easy to prove that $\left|u_{n+1}-v_{n+1}\right| \leq \frac{1}{2}\left|u_{n}-v_{n}\right|$ for all $n$, hence $u_{n}$ and $v_{n}$ converge to a common limit. This is the agm of $u$ and $v$, which we denote by $M(u, v)$. We will see later in a more general setting the well-known fact that the convergence is quadratic, hence the number of correct digits roughly doubles on each iteration step. (We refer to [3] for a systematic treatment of compound means like the agm.)

Lagrange and Gauss proved that

$$
I_{1}\left(a_{1}, a_{2}, a_{3}\right)=\frac{\pi}{M\left(\sqrt{a_{3}-a_{1}}, \sqrt{a_{3}-a_{2}}\right)}
$$

and

$$
I_{2}\left(a_{1}, a_{2}, a_{3}\right)=\frac{\pi}{M\left(\sqrt{a_{3}-a_{1}}, \sqrt{a_{2}-a_{1}}\right)} .
$$

For $d=4$ they showed that

$$
I_{1}\left(a_{1}, a_{2}, a_{3}, a_{4}\right)=I_{3}\left(a_{1}, a_{2}, a_{3}, a_{4}\right)=\frac{\pi}{M\left(\sqrt{a_{3,1} a_{4,2}}, \sqrt{a_{3,2} a_{4,1}}\right)}
$$

and

$$
I_{2}\left(a_{1}, a_{2}, a_{3}, a_{4}\right)=\frac{\pi}{M\left(\sqrt{a_{3,1} a_{4,2}}, \sqrt{a_{2,1} a_{4,3}}\right)},
$$

where $a_{i, j}:=a_{i}-a_{j}$. (Note that $a_{i, j}>0$ if $i>j$.) For $d=5$ and $d=6$, there exists a similar iterative algorithm due to Richelot (see $[4,20]$ for a modern account). The cases $d=7$ and $d=8$ were considered in the more general and abstract setting of Jacobians of genus 3 in $[7,16]$.

In this paper we deal with arbitrary $d$ and we give explicit iterative algorithms, extending the formulas by Lagrange and Gauss. To explain our result, we look at the hyperelliptic curve

$$
C: y^{2}=\left(x-a_{1}\right) \cdots\left(x-a_{d}\right) .
$$


$C$ is a compact Riemann surface of genus $g$, which is related to $d$ by $d=2 g+1$ if $d$ is odd and $d=2 g+2$ if $d$ is even. This Riemann surface is obtained by gluing two copies of $\mathbf{C} \cup\{\infty\}$ minus the "odd" cuts $\left[a_{1}, a_{2}\right],\left[a_{3}, a_{4}\right], \ldots,\left[a_{2 g+1}, a_{2 g+2}\right]$, where we set $a_{2 g+2}=\infty$ if $d=2 g+1$. The following picture illustrates the result for $g=3$, i.e. $d=7$ or $d=8$ :

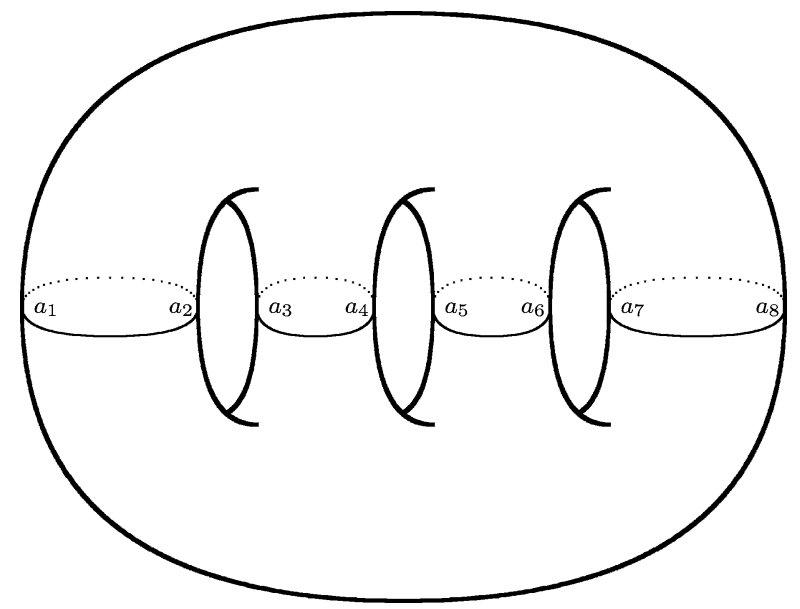

We see that the cases $d=2 g+1$ and $d=2 g+2$ are essentially equivalent. In fact, if $d$ is even then all branch points are finite, whereas $\infty$ is a branch point for odd $d$. Any Möbius transformation of the form

$$
x \mapsto \tilde{x}=\frac{c x+b}{x-a_{d}}, \quad x \mapsto \tilde{x}=\frac{a_{d} \tilde{x}+b}{\tilde{x}-c}
$$

makes $x=a_{d}$ correspond to $\tilde{x}=\infty$. Hence it suffices to consider the case where $d$ is even (or $d$ is odd). For example, for $d=4$ the transformation

$$
\tilde{x}=\frac{a_{4,2}\left(x-a_{1}\right)}{a_{2,1}\left(a_{4}-x\right)}
$$

identifies the quartic curve $y^{2}=\left(x-a_{1}\right)\left(x-a_{2}\right)\left(x-a_{3}\right)\left(x-a_{4}\right)$ with the cubic $\tilde{y}^{2}=\tilde{x}(\tilde{x}-1)(\tilde{x}-\lambda)$, where $\lambda:=\frac{a_{3,1} a_{4,2}}{a_{2,1} a_{4,3}}>1$. Note that $x=a_{1}, a_{2}, a_{3}, a_{4}$ correspond to $\tilde{x}=0,1, \lambda, \infty$, respectively. We find

$$
I_{1}\left(a_{1}, a_{2}, a_{3}, a_{4}\right)=\frac{1}{\sqrt{a_{2,1} a_{4,3}}} \int_{0}^{1} \frac{\mathrm{d} \tilde{x}}{\sqrt{\tilde{x}(\tilde{x}-1)(\tilde{x}-\lambda)}}
$$

and

$$
I_{2}\left(a_{1}, a_{2}, a_{3}, a_{4}\right)=\frac{1}{\sqrt{a_{2,1} a_{4,3}}} \int_{1}^{\lambda} \frac{\mathrm{d} \tilde{x}}{\sqrt{-\tilde{x}(\tilde{x}-1)(\tilde{x}-\lambda)}} .
$$

Using this transformation one sees that the formulas mentioned before for $d=3$ and $d=4$ are equivalent. 
More generally, we will consider integrals

$$
I_{j, k}\left(a_{1}, \ldots, a_{d}\right):=\int_{a_{j}}^{a_{j+1}} \frac{x^{k-1} \mathrm{~d} x}{\sqrt{\left|\left(x-a_{1}\right) \cdots\left(x-a_{d}\right)\right|}}
$$

for $k \geq 1$. These are closely related to the period matrix of the hyperelliptic curve $C$. In fact, Riemann showed that

$$
\psi_{k}:=x^{k-1} \frac{\mathrm{d} x}{2 y} \quad(k=1, \ldots, g)
$$

form a basis for the vector space of holomorphic 1-forms on $C$ (see for example $\left[12\right.$, p. 255]). We choose the standard symplectic basis $A_{1}, \ldots, A_{g}, B_{1}, \ldots, B_{g}$ for the homology of $C$ (see [19, p. 76]). We illustrate this for $g=3$ in the complex plane:

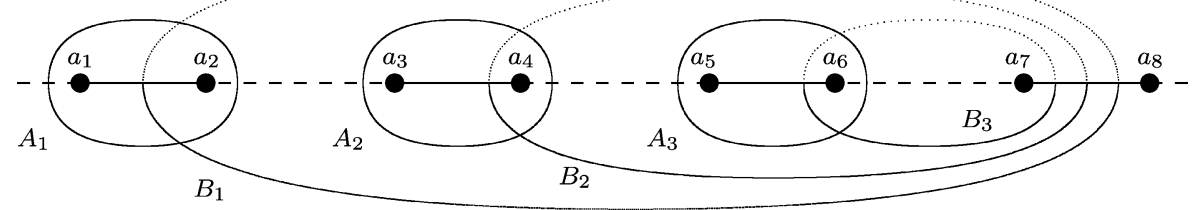

Note that $y$ has a single valued analytic continuation on these cycles. On the Riemann surface the cycles look like this:

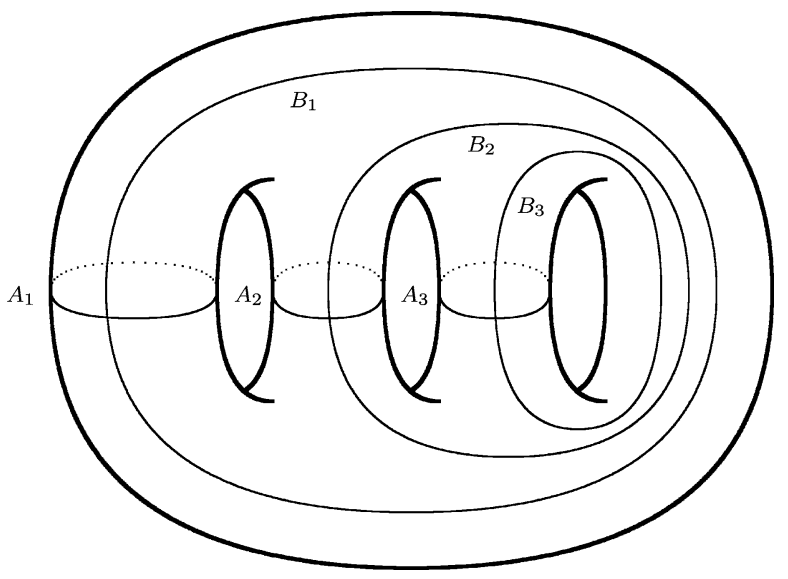

If we define $A_{g+1}$ in the obvious way, we see that $\sum_{j=1}^{g+1} A_{j}$ is homologous to 0 , whence

$$
\sum_{j=1}^{g+1}(-1)^{j} I_{2 j-1, k}=0
$$


for $k=1, \ldots, g$. The alternating sign is explained by the fact that the analytic continuation of $\sqrt{\left(x-a_{1}\right) \cdots\left(x-a_{d}\right)}$ changes sign between two cuts.

We now define two $g \times g$-matrices $\left(A_{k, j}\right)$ and $B=\left(B_{k, j}\right)$, where $1 \leq k, j \leq g$. If $d$ is odd, then $\left(x-a_{1}\right) \cdots\left(x-a_{d}\right)$ is positive on the intervals $\left[a_{2 j-1}, a_{2 j}\right]$ corresponding to the cycles $A_{j}$, hence

$$
\int_{A_{j}} \psi_{k}=(-1)^{j+1} I_{2 j-1, k}=: A_{k, j}
$$

If $d$ is even, then $\left(x-a_{1}\right) \cdots\left(x-a_{d}\right)$ is positive on the intervals corresponding to the cycles $B_{j}$. In fact,

$$
\int_{B_{j}} \psi_{k}=\sum_{\ell=j}^{g}(-1)^{\ell+1} I_{2 \ell, k}=: B_{k, j}
$$

Similarly we deal with the imaginary periods and we find that $C$ has period matrix

$$
\left(\int_{A_{j}} \psi_{k} \int_{B_{j}} \psi_{k}\right)= \begin{cases}(A i B) & \text { if } d \text { is odd } \\ (-i A B) & \text { if } d \text { is even }\end{cases}
$$

where $A$ and $B$ are the real $g \times g$-matrices $\left(A_{k, j}\right)$ and $B=\left(B_{k, j}\right)$, respectively. In each case, the normalized period matrix is $(1, i \Omega)$, where

$$
\Omega:=A^{-1} B \in \mathbf{R}^{g \times g} .
$$

Riemann's famous period relations imply that $\Omega$ is symmetric and positive definite (see [12, pp. 231/232]). We will check in Proposition 1 below that $\operatorname{det}(A)$ and $\operatorname{det}(B)$ do indeed have the same sign, namely $(-1)^{\lfloor g / 2\rfloor}$. In fact, we shall show that

$$
\operatorname{det}(A)=(-1)^{\lfloor g / 2\rfloor} \cdot \int_{x_{1}=a_{1}}^{a_{2}} \cdots \int_{x_{g}=a_{2 g-1}}^{a_{2 g}} \prod_{1 \leq k<j \leq g} \frac{\left(x_{j}-x_{k}\right) \mathrm{d} x_{1} \cdots \mathrm{d} x_{g}}{\sqrt{\left|f\left(x_{1}\right) \cdots f\left(x_{g}\right)\right|}}
$$

and

$$
\operatorname{det}(B)=(-1)^{\lfloor g / 2\rfloor} \cdot \int_{x_{1}=a_{2}}^{a_{3}} \cdots \int_{x_{g}=a_{2 g}}^{a_{2 g+1}} \prod_{1 \leq k<j \leq g} \frac{\left(x_{j}-x_{k}\right) \mathrm{d} x_{1} \cdots \mathrm{d} x_{g}}{\sqrt{\left|f\left(x_{1}\right) \cdots f\left(x_{g}\right)\right|}}
$$

The main result of this paper shows how to compute $\operatorname{det}(A), \operatorname{det}(B)$ and certain other minors using a generalized arithmetic-geometric mean. For genus $g$ we will have to take the generalized agm of $2^{g}$ numbers. For example, for $d=5$ one finds

$$
\operatorname{det}(A)=-\frac{\pi^{2}}{M\left(w_{1}, w_{2}, w_{3}, w_{4}\right)}
$$

where $w_{1}:=\sqrt{a_{3,1} a_{5,4} a_{5,2} a_{4,2}}, w_{2}:=\sqrt{a_{4,1} a_{5,3} a_{5,2} a_{3,2}}, w_{3}:=\sqrt{a_{3,2} a_{5,4} a_{5,1} a_{4,1}}$ and $w_{4}:=\sqrt{a_{4,2} a_{5,3} a_{5,1} a_{3,1}}$ and where the generalized agm $M(a, b, c, d)$ of four 
positive real numbers $a, b, c, d$ is defined as the common limit of the sequences

$$
\begin{aligned}
a_{n+1} & :=\frac{1}{4}\left(a_{n}+b_{n}+c_{n}+d_{n}\right), \\
b_{n+1} & :=\frac{1}{2}\left(\sqrt{a_{n} b_{n}}+\sqrt{c_{n} d_{n}}\right), \\
c_{n+1} & :=\frac{1}{2}\left(\sqrt{a_{n} c_{n}}+\sqrt{b_{n} d_{n}}\right), \\
d_{n+1} & :=\frac{1}{2}\left(\sqrt{a_{n} d_{n}}+\sqrt{b_{n} c_{n}}\right)
\end{aligned}
$$

with $a_{0}:=a, b_{0}:=b, c_{0}:=c$ and $d_{0}:=d$. This generalized agm was introduced by Borchardt in [2] and studied more recently in [8, 9, 14], also for complex arguments. Similarly, setting $a_{i, j, k}:=a_{i, j} a_{i, k} a_{j, k}$, we have

$$
\operatorname{det}(B)=-\frac{\pi^{2}}{M\left(\sqrt{a_{4,2} a_{5,3,1}}, \sqrt{a_{5,2} a_{4,3,1}}, \sqrt{a_{4,3} a_{5,2,1}}, \sqrt{a_{5,3} a_{4,2,1}}\right)} .
$$

We can also compute

$$
\operatorname{det}\left(\begin{array}{ll}
A_{0,1} & B_{0,2} \\
A_{1,1} & B_{1,2}
\end{array}\right)=-\frac{\pi^{2}}{M\left(\sqrt{a_{4,1} a_{5,3,2}}, \sqrt{a_{4,2} a_{5,3,1}}, \sqrt{a_{5,1} a_{4,3,2}}, \sqrt{a_{5,2} a_{4,3,1}}\right)}
$$

and

$$
\operatorname{det}\left(\begin{array}{ll}
A_{0,2} & B_{0,1} \\
A_{1,2} & B_{1,1}
\end{array}\right)=\frac{\pi^{2}}{M\left(\sqrt{a_{3,2} a_{5,4,1}}, \sqrt{a_{5,3} a_{4,2,1}}, \sqrt{a_{5,2} a_{4,3,1}}, \sqrt{a_{5,4} a_{3,2,1}}\right)} .
$$

We leave it to the reader to find the pattern. (Draw a picture of the branch points and the cycles $A_{1}, A_{2}, B_{1}, B_{2}$ in the complex plane!) However, we cannot compute the remaining two minors $\operatorname{det}\left(\begin{array}{ll}A_{0,1} & B_{0,1} \\ A_{1,1} & B_{1,1}\end{array}\right)$ and $\operatorname{det}\left(\begin{array}{ll}A_{0,2} & B_{0,2} \\ A_{1,2} & B_{1,2}\end{array}\right)$ this way. Note that these two minors are "non-Lagrangian" in the sense that the intersection numbers $A_{1} \cdot B_{1}$ and $A_{2} \cdot B_{2}$ do not vanish.

As mentioned above, one can use a Möbius transformation to deduce the corresponding results for $d=6$. One finds

$$
\operatorname{det}(A)=-\frac{\pi^{2}}{M\left(\sqrt{a_{6,3,1} a_{5,4,2}}, \sqrt{a_{6,4,1} a_{5,3,2}}, \sqrt{a_{6,3,2} a_{5,4,1}}, \sqrt{a_{6,4,2} a_{5,3,1}}\right)} .
$$

Note that the corresponding result for $d=5$ is obtained by simply omitting the index "6". The same holds for the other minors, e.g.

$$
\operatorname{det}(B)=-\frac{\pi^{2}}{M\left(\sqrt{a_{6,4,2} a_{5,3,1}}, \sqrt{a_{6,5,2} a_{4,3,1}}, \sqrt{a_{6,4,3} a_{5,2,1}}, \sqrt{a_{6,5,3} a_{4,2,1}}\right)} .
$$

Since the generalized agm converges quadratically, it provides an efficient way to compute the corresponding minors of the period matrix of the hyperelliptic curve 
$y^{2}=\left(x-a_{1}\right) \cdots\left(x-a_{2 g+2}\right)$. Conversely, one might ask which generalized agm's appear this way. Since $2^{g}>2 g+1$ for $g \geq 3$ it is clear that most $2^{g}$-tuples do not arise this way. For $g=2, d=5$ one has to deal with the map $\left(a_{1}, a_{2}, a_{3}, a_{4}, a_{5}\right) \mapsto$ $(x, y, z, t)$, where

$$
\begin{aligned}
x & :=w_{1}^{2}=\left(a_{3}-a_{1}\right)\left(a_{5}-a_{4}\right)\left(a_{5}-a_{2}\right)\left(a_{4}-a_{2}\right), \\
y & :=w_{2}^{2}=\left(a_{4}-a_{1}\right)\left(a_{5}-a_{3}\right)\left(a_{5}-a_{2}\right)\left(a_{3}-a_{2}\right), \\
z & :=w_{3}^{2}=\left(a_{3}-a_{2}\right)\left(a_{5}-a_{4}\right)\left(a_{5}-a_{1}\right)\left(a_{4}-a_{1}\right), \\
t & :=w_{4}^{2}=\left(a_{4}-a_{2}\right)\left(a_{5}-a_{3}\right)\left(a_{5}-a_{1}\right)\left(a_{3}-a_{1}\right) .
\end{aligned}
$$

As only differences of $a_{j}$ 's appear, we may as well put $a_{5}=0$ and we end up with a map $\Psi: \mathbf{C}^{4} \rightarrow \mathbf{C}^{4}$. The geometry of this map is quite involved: it is not surjective, as one readily checks that $(1,0,0,0)$ is not in the image. On the other hand the map is dominant and a general 4-tuple like $(1,2,3,4)$ has 16 preimages. The discriminant of the map $\Psi$ has the form $y z \operatorname{th}(x, y, z, t)$, where $h(x, y, z, t)$ is the homogeneous quartic polynomial

$$
\left((x+y+z+t)^{2}-4(x y+x z+x t+y z+y t+z t)\right)^{2}-64 x y z t
$$

whose vanishing describes a Steiner roman surface in projective three-space. All these facts can be checked with a computer algebra system like SINGULAR. The map $\Psi$ is also considered in [17]. (Unfortunately, however, Theorem 1 in [17] and the formulas on p. 275 of [17] are wrong.)

The structure of this paper is as follows. First, in Sect. 2, we give two classical proofs for the case $g=1$ : one using a miraculous substitution and another using theta constants. We explain the substitution in terms of isogenies and apply this to compute elliptic periods in the "rhombic case", i.e. integrals of the form $\int_{p}^{\infty} \mathrm{d} x / \sqrt{(x-p)\left(x^{2}+p x+q\right)}$ with $p^{2}-4 q<0$. In the third section we will generalize the second proof to arbitrary genus $g$. Section four contains a numerical example for genus 3 . In the final section we deal with the convergence properties of the generalized agm.

\section{Elliptic case: $g=1$}

An elliptic curve is a curve of genus 1 and the corresponding integrals are called elliptic periods. They are related to the length of an ellipse, which explains the adjective 'elliptic' (see for instance [1]).

\subsection{Two classical approaches}

We will prove the formulas for the elliptic periods twice: first using a clever substitution and second using theta constants. Both ideas are due to Gauss (see [5]). We start with an elliptic integral written in a slightly different form. 
Theorem 1 (Lagrange (1784), Gauss (1799)) If $a, b>0$ then

$$
\int_{0}^{\frac{1}{2} \pi} \frac{\mathrm{d} \vartheta}{\sqrt{a^{2} \cos ^{2}(\vartheta)+b^{2} \sin ^{2}(\vartheta)}}=\frac{\frac{1}{2} \pi}{M(a, b)} .
$$

Proof The substitution $\sin \vartheta=\frac{2 a \sin \varphi}{(a+b)+(a-b) \sin ^{2} \varphi}$ yields $I(a, b)=I\left(a^{\prime}, b^{\prime}\right)$, where $a^{\prime}:=\frac{1}{2}(a+b)$ and $b^{\prime}:=\sqrt{a b}$. Hence $I(a, b)=I\left(a^{\prime}, b^{\prime}\right)=I\left(a^{\prime \prime}, b^{\prime \prime}\right)=\cdots=$ $I(M, M)=(2 \pi M)^{-1}$, since the integrand converges uniformly to the constant function $M$.

Corollary 1 If $a_{1}<a_{2}<a_{3}$ then

$$
\int_{a_{1}}^{a_{2}} \frac{\mathrm{d} x}{\sqrt{\left(x-a_{1}\right)\left(x-a_{2}\right)\left(x-a_{3}\right)}}=\frac{\pi}{M\left(\sqrt{a_{3}-a_{1}}, \sqrt{a_{3}-a_{2}}\right)} .
$$

Proof Substitution of $x=a_{1}+\left(a_{2}-a_{1}\right) \sin ^{2}(\vartheta)$ reduces this to the theorem.

Below, we will give an explanation for the remarkable substitution $\sin \vartheta=$ $\frac{2 a \sin \varphi}{(a+b)+(a-b) \sin ^{2} \varphi}$ used in the proof above. Our generalization to the hyperelliptic case (i.e. $g>1$ ) starts from another line of attack using theta functions, which we will now explain. We start with the elliptic curve

$$
E: \quad y^{2}=\left(x-a_{1}\right)\left(x-a_{2}\right)\left(x-a_{3}\right)
$$

satisfying $a_{1}<a_{2}<a_{3}$. Its period lattice is generated by $\omega_{1}:=I_{1}\left(a_{1}, a_{2}, a_{3}\right)$ and $\omega_{2}:=i I_{2}\left(a_{1}, a_{2}, a_{3}\right)$. We set $\tau:=\omega_{2} / \omega_{1}$ and $q:=e^{\pi i \tau}$. Note that $0<q<1$. We define the theta constants

$$
\vartheta_{0}(\tau):=\sum_{n=-\infty}^{\infty} q^{n^{2}}, \quad \vartheta_{1}(\tau):=\sum_{n=-\infty}^{\infty}(-1)^{n} q^{n^{2}} .
$$

Note that these constants are real numbers. Then one can show that

$$
\pi \vartheta_{0}(\tau)^{2}=\omega_{1} \sqrt{a_{3,1}}, \quad \pi \vartheta_{1}(\tau)^{2}=\omega_{1} \sqrt{a_{3,2}}
$$

(see [4, p. 8]). These identities were generalized to arbitrary genus by Thomae in [21] and we will refer to them as Thomae formulas. Furthermore, using the $q$-expansions above one easily checks that

$$
\begin{aligned}
& \vartheta_{0}^{2}(2 \tau)=\frac{1}{2}\left(\vartheta_{0}^{2}(\tau)+\vartheta_{1}^{2}(\tau)\right), \\
& \vartheta_{1}^{2}(2 \tau)=\vartheta_{0}(\tau) \vartheta_{1}(\tau)
\end{aligned}
$$

Since $\vartheta_{0}(\tau)$ is positive for $\tau$ on the positive imaginary axis, the second formula shows that the same holds for $\vartheta_{1}(\tau)$. Now we claim that

$$
M\left(\vartheta_{0}^{2}(\tau), \vartheta_{1}^{2}(\tau)\right)=1
$$


Indeed, applying the iterative definition of the agm starting with $a_{0}=\vartheta_{0}^{2}(\tau)$ and $b_{0}=\vartheta_{1}^{2}(\tau)$, we find

$$
a_{1}=\frac{1}{2}\left(a_{0}+b_{0}\right)=\vartheta_{0}^{2}(2 \tau) \quad \text { and } \quad b_{1}=\sqrt{a_{0} b_{0}}=\vartheta_{1}^{2}(2 \tau) .
$$

Iteration leads to

$$
a_{n}=\vartheta_{0}^{2}\left(2^{n} \tau\right) \text { and } \quad b_{n}=\vartheta_{1}^{2}\left(2^{n} \tau\right)
$$

It remains to note that $q$ tends to 0 as $n$ tends to infinity, hence $a_{n}$ and $b_{n}$ tend to 1 . Together with the Thomae formulas, we find

$$
\pi=M\left(\pi \vartheta_{0}(\tau)^{2}, \pi \vartheta_{1}(\tau)^{2}\right)=M\left(\omega_{1} \sqrt{a_{3,1}}, \omega_{1} \sqrt{a_{3,2}}\right)=\omega_{1} M\left(\sqrt{a_{3,1}}, \sqrt{a_{3,2}}\right) .
$$

\subsection{An isogeny of degree 2}

Recall that $E \cong \mathbf{C} / \Lambda$, where $\Lambda=\mathbf{Z} \oplus \tau \mathbf{Z}$. Replacing $\tau$ with $2 \tau$ corresponds to replacing $E$ with $E^{\prime}:=\mathbf{C} / \Lambda^{\prime}$, where $\Lambda^{\prime}:=\mathbf{Z} \oplus 2 \tau \mathbf{Z}$. The inclusion $\Lambda^{\prime} \subset \Lambda$ defines an isogeny $E^{\prime} \rightarrow E$ of degree 2 . We will write down this isogeny explicitly in terms of the coefficients $a_{1}, a_{2}, a_{3}$ and we will see that this explains the miraculous substitution $\sin \vartheta=\frac{2 u \sin \varphi}{(u+v)+(u-v) \sin ^{2} \varphi}$.

Gauss used this substitution to show that

$$
I(u, v):=\int_{0}^{\frac{1}{2} \pi}\left(u^{2} \cos ^{2}(\vartheta)+v^{2} \sin ^{2}(\vartheta)\right)^{-1 / 2} \mathrm{~d} \vartheta
$$

is invariant under

$$
(u, v) \mapsto\left(u^{\prime}, v^{\prime}\right):=\left(\frac{1}{2}(u+v), \sqrt{u v}\right) .
$$

To compare this to our elliptic integrals, we recall that

$$
I_{1}\left(a_{1}, a_{2}, a_{3}\right)=\int_{a_{1}}^{a_{2}} \frac{\mathrm{d} x}{\sqrt{\left(x-a_{1}\right)\left(x-a_{2}\right)\left(x-a_{3}\right)}}=2 I(u, v),
$$

where $u=\sqrt{a_{3,1}}$ and $v=\sqrt{a_{3,2}}$. If we assume that $a_{1}+a_{2}+a_{3}=0$ and set

$$
\begin{aligned}
& a_{1}^{\prime}:=\frac{1}{3}\left(-2 u^{\prime 2}+v^{\prime 2}\right)=-\frac{1}{2} a_{3} \\
& a_{2}^{\prime}:=\frac{1}{3}\left(u^{\prime 2}-2 v^{\prime 2}\right)=\frac{1}{4} a_{3}-\frac{1}{2} \sqrt{a_{3,1} a_{3,2}} \\
& a_{3}^{\prime}:=\frac{1}{3}\left(u^{\prime 2}+v^{\prime 2}\right)=\frac{1}{4} a_{3}+\frac{1}{2} \sqrt{a_{3,1} a_{3,2}},
\end{aligned}
$$

then $a_{1}^{\prime}+a_{2}^{\prime}+a_{3}^{\prime}=0, a_{1}^{\prime}<a_{2}^{\prime}<a_{3}^{\prime}$ and $\left(u^{\prime}, v^{\prime}\right)=\left(\sqrt{a_{3,1}^{\prime}}, \sqrt{a_{3,2}^{\prime}}\right)$. Hence the equality $I(u, v)=I\left(u^{\prime}, v^{\prime}\right)$ is equivalent to $I_{1}\left(a_{1}, a_{2}, a_{3}\right)=I_{1}\left(a_{1}^{\prime}, a_{2}^{\prime}, a_{3}^{\prime}\right)$. One can check that 
under the correspondence of Corollary 1 , the substitution $\sin \vartheta=\frac{2 u \sin \varphi}{(u+v)+(u-v) \sin ^{2} \varphi}$ is equivalent to

$$
\left(x^{\prime}, y^{\prime}\right) \mapsto(x, y)=\left(x^{\prime}+\frac{a_{3,1}^{\prime} a_{3,2}^{\prime}}{x^{\prime}-a_{1}^{\prime}},\left(1-\frac{a_{3,1}^{\prime} a_{3,2}^{\prime}}{\left(x^{\prime}-a_{1}^{\prime}\right)^{2}}\right) y^{\prime}\right)
$$

from $E^{\prime}: y^{\prime 2}=\left(x-a_{1}^{\prime}\right)\left(x-a_{2}^{\prime}\right)\left(x-a_{3}^{\prime}\right)$ to $E: y^{2}=\left(x-a_{1}\right)\left(x-a_{2}\right)\left(x-a_{3}\right)$. One can check that

$$
\frac{\mathrm{d} x}{\sqrt{\left(x-a_{1}\right)\left(x-a_{2}\right)\left(x-a_{3}\right)}}=\frac{\mathrm{d} x^{\prime}}{\sqrt{\left(x^{\prime}-a_{1}^{\prime}\right)\left(x^{\prime}-a_{2}^{\prime}\right)\left(x^{\prime}-a_{3}^{\prime}\right)}},
$$

whence $I_{1}\left(a_{1}, a_{2}, a_{3}\right)=I_{1}\left(a_{1}^{\prime}, a_{2}^{\prime}, a_{3}^{\prime}\right)$. The same substitution can be used to show that $I_{2}\left(a_{1}, a_{2}, a_{3}\right)=\frac{1}{2} I_{2}\left(a_{1}^{\prime}, a_{2}^{\prime}, a_{3}^{\prime}\right)$.

Note that $\left(a_{1}^{\prime}, 0\right)$ is mapped to the point at infinity, whereas $\left(a_{2}^{\prime}, 0\right)$ and $\left(a_{3}^{\prime}, 0\right)$ are mapped to $\left(a_{3}, 0\right)$. In fact, $E^{\prime} \rightarrow E$ is an isogeny of degree 2 that mods out the 2-torsion point $\left(a_{1}^{\prime}, 0\right)$ of $E^{\prime}$. In terms of the period lattices we find that the imaginary periods of $E^{\prime}$ are twice the imaginary periods of $E$, whereas the real periods coincide. So we see that the doubling of $\tau$ corresponds to the substitution $\sin \vartheta=\frac{2 u \sin \varphi}{(u+v)+(u-v) \sin ^{2} \varphi}$.

\subsection{Application to rhombic elliptic periods}

We can use these ideas to compute rhombic elliptic periods, i.e. integrals of the form

$$
\int_{p}^{\infty} \frac{\mathrm{d} x}{\sqrt{(x-p)\left(x^{2}+p x+q\right)}}
$$

with $\Delta:=p^{2}-4 q<0$.

Theorem 2 If $p^{2}-4 q<0$ then $a_{1}=p-2 \sqrt{2 p^{2}+q}, a_{2}=-2 p, a_{3}=p+$ $2 \sqrt{2 p^{2}+q}$ satisfy $a_{1}<a_{2}<a_{3}$ and

$$
\begin{aligned}
\int_{p}^{\infty} \frac{\mathrm{d} x^{\prime}}{\sqrt{\left(x^{\prime}-p\right)\left(x^{\prime 2}+p x^{\prime}+q\right)}} & =2 \int_{a_{1}}^{a_{2}} \frac{\mathrm{d} x}{\sqrt{\left(x-a_{1}\right)\left(x-a_{2}\right)\left(x-a_{3}\right)}} \\
& =\frac{2 \pi}{M\left(\sqrt{a_{2,1}}, \sqrt{a_{3,2}}\right)} .
\end{aligned}
$$

Proof The inequalities $a_{1}<a_{2}$ and $a_{2}<a_{3}$ are clear. The idea is to mod out the real 2-torsion point $(p, 0)$ of

$$
E^{\prime}: \quad y^{2}=(x-p)\left(x^{2}+p x+q\right) .
$$

Note that the period lattice of $E^{\prime}$ is rhombic, i.e. if $\omega_{1}$ and $\omega_{2}$ are the first periods on the positive real and imaginary axis, respectively, then the period lattice $\Lambda^{\prime}$ is generated by $\omega_{1}$ and $\frac{1}{2}\left(\omega_{1}+\omega_{2}\right)$. Hence $\Lambda:=\mathbf{Z} \omega_{1} \oplus \mathbf{Z} \omega_{2}$ is a sublattice of index 2 . The 
corresponding elliptic curve is $E:=E^{\prime} /(p, 0)$. Since its period lattice is rectangular, it can be written as

$$
E: \quad y^{2}=\left(x-a_{1}\right)\left(x-a_{2}\right)\left(x-a_{3}\right)
$$

with real branch points $b_{1}, b_{2}, b_{3}$. Setting $a_{1}^{\prime}:=p$ and $a_{2,3}^{\prime}:=-\frac{1}{2} p \pm \frac{1}{2} i \sqrt{-\Delta}$ and reversing the formulas mentioned above, we find $a_{3}=2 p, a_{2}=p+2 \sqrt{2 p^{2}+q}$ and $a_{1}=p-2 \sqrt{2 p^{2}+q}$. It remains to note that we have interchanged $a_{2}$ and $a_{3}$ in the statement of the theorem to conform to our convention that $a_{2}<a_{3}$.

Example $1 p=0$ and $q=1$, hence $a_{3}=0, a_{2}=2, a_{1}=-2$ and

$$
\begin{aligned}
\int_{0}^{\infty} \frac{\mathrm{d} x^{\prime}}{\sqrt{x^{\prime}\left(x^{\prime 2}+1\right)}} & =2 \int_{0}^{\infty} \frac{\mathrm{d} x}{\sqrt{x\left(x^{2}-4\right)}}=2 \int_{-2}^{0} \frac{\mathrm{d} x}{\sqrt{x\left(x^{2}-4\right)}} \\
& =\frac{2 \pi}{M(\sqrt{4}, \sqrt{2})}=\frac{2 \pi}{1.694426170 \ldots}=3.708149355 \ldots
\end{aligned}
$$

The transformation boils down to $x=x^{\prime}+\frac{1}{x^{\prime}}$. The period in this example is a factor $\sqrt{2}$ smaller than

$$
2 \int_{-1}^{1} \frac{\mathrm{d} x}{\sqrt{1-x^{4}}}=\frac{2 \pi}{M(\sqrt{2}, 1)}=5.244115109 \ldots
$$

which is the length of the lemniscate $r^{2}=\cos (2 \theta)$, as calculated by Gauss. It is the computation of the length of the lemniscate in 1799 which led Gauss to his theory of the arithmetic-geometric mean. For more historical details see [5, 6].

\section{Main theorem}

The second proof in the section about the elliptic case using theta constants generalizes to the hyperelliptic case. The main ingredients are again the doubling formula for theta constants and the Thomae formulas, which relate theta constants to hyperelliptic integrals and branch points.

3.1 Doubling formula and generalized arithmetic-geometric mean

We first define theta constants. We denote Siegel upper half space, consisting of all symmetric $g \times g$-matrices with positive definite imaginary part, by $\mathfrak{H}_{g}$.

Definition For $\tau \in \mathfrak{H}_{g}$ and $\beta \in(\mathbf{Z} / 2 \mathbf{Z})^{g}=\mathbf{F}_{2}^{g}$ we define the theta constant

$$
\vartheta_{\beta}(\tau):=\sum_{m \in \mathbf{Z}^{g}} \exp \left(\pi i m^{T} \tau m+\pi i m^{T} \beta\right) .
$$


Our $\vartheta_{\beta}(\tau)$ is traditionally denoted by $\vartheta\left[\begin{array}{c}0 \\ \frac{1}{2} \beta\end{array}\right](\tau, 0)$ (see [18, p. 123] or [13]). Note that $\vartheta_{\beta}(i \Omega)$ is real if $\Omega$ is. Note also that $\vartheta_{\beta}\left(2^{n} \tau\right) \rightarrow 1$ as $n \rightarrow \infty$.

Now we can state the doubling formula for (even) theta constants.

Theorem 3 For $\tau \in \mathfrak{H}_{g}$ and $\beta \in(\mathbf{Z} / 2 \mathbf{Z})^{g}=\mathbf{F}_{2}^{g}$ we have

$$
\vartheta_{\beta}^{2}(2 \tau)=\frac{1}{2^{g}} \sum_{\gamma \in \mathbf{F}_{2}^{g}} \vartheta_{\gamma}(\tau) \vartheta_{\beta+\gamma}(\tau) .
$$

Proof This is a special case of Theorem 2 in [13, p. 139].

From this we deduce the following definition of the generalized algebraicgeometric mean for a $2^{g}$-tuple of positive real numbers. More precisely, we will define the generalized agm of a positive function $v: V \rightarrow \mathbf{R}^{+}$where $V$ is a vector space of dimension $g$ over $\mathbf{F}_{2}$. We set $v_{0}:=v$ and

$$
v_{n+1}(\beta):=\frac{1}{2^{g}} \sum_{\gamma \in V} \sqrt{v_{n}(\gamma) v_{n}(\beta+\gamma)} .
$$

We can view $v_{n}$ as a sequence of functions on $V$ or as a $2^{g}$-tuple of sequences $v_{n}(\beta)$, one for each $\beta \in V$. In the next section (see Theorems 6 and 7) we will prove that these $2^{g}$ sequences converge quadratically to a common limit. Equivalently, the sequence $v_{n}$ converges quadratically to a constant function on $V$.

Definition The generalized arithmetic-geometric mean $M(v)$ of $v=v_{0}$ is defined as the common limit of the $2^{g}$ defining sequences $v_{n}(\beta)$.

Remark 1 This generalized arithmetic-geometric mean was also considered in [8] and [9] where in particular the behavior for complex arguments was studied. Note that for $g \geq 3$ the generalized agm depends on the parametrization of the $2^{g}$-tuple. More precisely, if $\sigma$ is a permutation of $V$, then in general one has $M(v) \neq M(v \circ \sigma)$.

An important special case is $V=\mathbf{F}_{2}^{g}$ and $v(\beta)=\vartheta_{\beta}^{2}(\tau)$ for fixed $\tau \in \mathfrak{H}_{g}$. Recall that $\vartheta_{\beta}(\tau)$ is real, since $\Omega \in \mathbf{R}^{g \times g}$, hence $\vartheta_{\beta}^{2}(\tau) \geq 0$. In fact, the Thomae formulas below imply that $\vartheta_{\beta}(\tau) \neq 0$, hence $\vartheta_{\beta}^{2}(\tau)>0$.

Lemma 1 Fix $\tau \in \mathfrak{H}_{g}$ and set $v(\beta):=\vartheta_{\beta}^{2}(\tau)$ for $\beta \in \mathbf{F}_{2}^{g}$. Then $M(v)=1$.

Proof By the doubling formula and the definition of the generalized agm we find that $M(v)=M\left(\beta \mapsto \vartheta_{\beta}^{2}\left(2^{n} \tau\right)\right)$ for $n=1,2,3, \ldots$ It remains to note that $\vartheta_{\beta}^{2}\left(2^{n} \tau\right) \rightarrow 1$ as $n \rightarrow \infty$. 


\subsection{Thomae formulas}

The Thomae formulas are the second main ingredient for the relation between hyperelliptic integrals and the generalized agm. Let

$$
y^{2}=\left(x-a_{1}\right) \cdots\left(x-a_{d}\right)
$$

be a real hyperelliptic curve satisfying $a_{1}<\cdots<a_{d}$. We have $d=2 g+1$ or $d=$ $2 g+2$, where $g$ is the genus. In the introduction we defined period matrices $A, B$ and $i \Omega$.

We say that $S \subseteq\{1, \ldots, 2 g+2\}$ is an $A$-subset if $S$ is a union of subsets of the form $\{2 j-1,2 j\}$. For an $A$-subset $S$ we set

$$
\beta_{j}:=\# S \cap\{2 j, \ldots, 2 g+1\} \bmod 2
$$

for $j=1, \ldots, g$.

Let $\mathbf{A}$ be the set of $A$-subsets modulo $A:=A^{c}:=\{1, \ldots, 2 g+2\} \backslash S$. This set is a vector space of dimension $g$ over $\mathbf{F}_{2}$ with the symmetric difference "o" as addition and the empty set as zero element. The map

$$
\beta=\left(\beta_{1}, \ldots, \beta_{g}\right): \quad \mathbf{A} \rightarrow \mathbf{F}_{2}^{g}
$$

is a vector space isomorphism. The standard basis of $\mathbf{F}_{2}^{g}$ corresponds to the basis $\{1,2\}, \ldots,\{2 g-1,2 g\}$ of $\mathbf{A}$.

Let $w: \mathbf{A} \rightarrow \mathbf{R}^{+}$be a family of positive real numbers parametrized by $\mathbf{A}$. Note that $M(w)$ is the common limit of the sequences $\left(w_{n}(S)\right)_{n=0}^{\infty}$ defined by the iteration

$$
w_{n+1}(S):=\frac{1}{2^{g}} \sum_{T \in \mathbf{A}} \sqrt{w_{n}(T) w_{n}(S \circ T)}
$$

and $w_{0}:=w$.

Theorem 4 (Thomae) Let $\tau=i \Omega$ be the period matrix of the hyperelliptic curve

$$
y^{2}=\left(x-a_{1}\right) \cdots\left(x-a_{2 g+2}\right)
$$

satisfying $a_{1}<\cdots<a_{2 g+2}<\infty$. For any subset $T \subseteq\{1, \ldots, 2 g+2\}$, we set

$$
\Pi_{T}:=\prod_{\substack{j>k, j, k \in T}}\left(a_{j}-a_{k}\right)>0 .
$$

If $S$ is an A-subset, then we have the Thomae formula

$$
\pi^{g} \vartheta_{\beta(S)}^{2}(\tau)=|\operatorname{det}(A)| \sqrt{\Pi_{S \circ U} \Pi_{S \circ G}},
$$

where $U=\{1,3, \ldots, 2 g+1\}$ and $G=\{2,4, \ldots, 2 g+2\}$ denote the subsets of odd and even elements, respectively. 
Proof This was proven by Thomae in 1870 (see [21]). For a modern account, see [10, p. 46] and [19, pp. 120/121]. Note that our $\left[\begin{array}{c}0 \\ \frac{1}{2} \beta(S)\end{array}\right]$ equals Mumford's $\eta_{S}$ (see $\left[19\right.$, p. 88]), hence $\vartheta_{\beta(S)}=\vartheta\left[\eta_{S}\right]$.

\subsection{Main theorem}

After these preliminaries, the main theorem follows easily.

Theorem 5 For a real hyperelliptic curve $y^{2}=\left(x-a_{1}\right) \cdots\left(x-a_{2 g+2}\right)$ we have

$$
|\operatorname{det}(A)|=\frac{\pi^{g}}{M\left(S \mapsto \sqrt{\Pi_{S \circ U} \Pi_{S \circ G}}\right)} .
$$

Proof This follows from the Thomae formulas, the doubling formula for $\vartheta$ and the fact that $\vartheta_{\beta}\left(2^{n} \tau\right) \rightarrow 1$ as $n \rightarrow \infty$ :

$$
\begin{aligned}
\pi^{g} & =\pi^{g} \cdot M\left(\beta \mapsto \vartheta_{\beta}^{2}(\tau)\right) \\
& =M\left(S \mapsto \pi^{g} \vartheta_{\beta(S)}^{2}(\tau)\right) \\
& =|\operatorname{det}(A)| \cdot M\left(S \mapsto \sqrt{\Pi_{S \circ U} \Pi_{S \circ G}}\right) .
\end{aligned}
$$

Remark 2 In the examples in the introduction we have seen how to translate this to the case $d=2 g+1$.

For completeness, we compute the sign of the determinants of $A$ and $B$.

Proposition $1 \operatorname{det}(A)$ and $\operatorname{det}(B)$ have sign $(-1)^{\lfloor g / 2\rfloor}$. In particular, $\operatorname{det}(\Omega)>0$.

Proof We have $\operatorname{det}(B)=\operatorname{det}\left((-1)^{j+1} I_{2 j, k}\right)=(-1)^{\lfloor g / 2\rfloor} \operatorname{det}\left(I_{2 j, k}\right)$, where $j$ and $k$ run from 1 through $g$. It remains to note that the Vandermonde determinant

$$
\begin{aligned}
\operatorname{det}\left(I_{2 j-1, k}\right)_{1 \leq j, k \leq g} & =\operatorname{det}\left(\int_{x_{j}=a_{2 j-1}}^{a_{2 j}} \frac{x_{j}^{k-1} \mathrm{~d} x_{j}}{\sqrt{\left|f\left(x_{j}\right)\right|}}\right)_{1 \leq j, k \leq g} \\
& =\int_{D} \prod_{1 \leq k<j \leq g} \frac{\left(x_{j}-x_{k}\right) \mathrm{d} x_{1} \cdots \mathrm{d} x_{g}}{\sqrt{\left|f\left(x_{1}\right) \cdots f\left(x_{g}\right)\right|}},
\end{aligned}
$$

where $D:=\prod_{j=1}^{g}\left[a_{2 j}, a_{2 j+1}\right]$, is positive. The computation of the sign of $\operatorname{det}(A)$ is analogous.

\section{Numerical example}

We consider the curve $y^{2}=\left(x-a_{1}\right) \cdots\left(x-a_{7}\right)$ with $a_{j}:=j^{2}$ for $j=1, \ldots, 7$ of genus 3. Using Maple (see the Appendix) with 10 digits accuracy we find 


$$
A \approx\left(\begin{array}{ccc}
0.001835389715 & -0.003233181628 & 0.001610591063 \\
0.004924225388 & -0.03980336598 & 0.04745548964 \\
0.01523975237 & -.5102224404 & 1.421915838
\end{array}\right)
$$

and

$$
\Omega \approx\left(\begin{array}{lll}
1.710058007 & 0.879176705 & 0.476414775 \\
0.879176703 & 1.372004622 & 0.565962196 \\
0.476414773 & 0.565962195 & 1.035264177
\end{array}\right)
$$

Note that $\Omega$ is indeed symmetric (up to 8 digits) and positive definite! Computing with 20 digits accuracy yields

$$
\operatorname{det}(A) \approx-0.000042207216090372187073 \text {. }
$$

We will now check that the generalized agm gives the same numerical result for $\operatorname{det}(A)$. There are $16 A$-subsets $S \subseteq\{1, \ldots, 8\}$ which give rise to 8 classes $[S] \in$ $V \cong \mathbf{F}_{2}^{3}$. We compute $w([S]):=\sqrt{\Pi_{S \circ U} \Pi_{S \circ G}}$ for each $[S] \in V$. The classes of $S_{1}:=\{1,2\}, S_{2}:=\{3,4\}$ and $S_{3}:=\{5,6\}$ form a basis of $V$; this way we identify $V$ with $\mathbf{F}_{2}^{3}$. As before, let $a_{i, j, k}=\left(a_{i}-a_{j}\right)\left(a_{i}-a_{k}\right)\left(a_{k}-a_{\ell}\right)$ and let $a_{i, j, k, \ell}$ denote the product of the six differences $a_{i}-a_{j}, \ldots, a_{k}-a_{\ell}$. We find

$$
\begin{aligned}
& w(0,0,0):=\sqrt{a_{7,5,3,1} \cdot a_{6,4,2}}=737280 \sqrt{2}, \\
& w(1,0,0):=\sqrt{a_{7,5,3,2} \cdot a_{6,4,1}}=7372800 \sqrt{210}, \\
& w(0,1,0):=\sqrt{a_{7,5,4,1} \cdot a_{6,3,2}}=17694720 \sqrt{15}, \\
& w(0,0,1):=\sqrt{a_{7,6,3,1} \cdot a_{5,4,2}}=13271040 \sqrt{14}, \\
& w(1,1,0):=\sqrt{a_{7,5,4,2} \cdot a_{6,3,1}}=8847360 \sqrt{105}, \\
& w(1,0,1):=\sqrt{a_{7,6,3,2} \cdot a_{5,4,1}}=26542080 \sqrt{5}, \\
& w(0,1,1):=\sqrt{a_{7,6,4,1} \cdot a_{5,3,2}}=2949120 \sqrt{210}, \\
& w(1,1,1):=\sqrt{a_{7,6,4,2} \cdot a_{5,3,1}}=23592960 \sqrt{6} .
\end{aligned}
$$

Four iterations of the agm-algorithm yield twenty-one correct digits:

$$
\frac{\pi^{3}}{M(w)}=\frac{\pi^{3}}{734620.274739527460876}=0.0000422072160903721870729,
$$

which does indeed agree with the direct computation of $|\operatorname{det}(A)|$ !

\section{Quadratic convergence of the generalized agm}

In this final section we prove the quadratic convergence of the generalized agm. First we prove convergence. 
Theorem 6 The sequence $v_{n}$ converges to a constant function on $V$. Equivalently, the $2^{g}$ sequences $v_{n}(\beta)$ converge to a common limit.

Proof For notational convenience we consider the case $g=3$; the general case is completely analogous. We have 8 sequences $a_{n}, \ldots, h_{n}$, where

$$
\begin{aligned}
& a_{n+1}=\frac{1}{8}\left(a_{n}+\cdots+h_{n}\right), \\
& b_{n+1}=\frac{1}{4}\left(\sqrt{a_{n} b_{n}}+\cdots+\sqrt{g_{n} h_{n}}\right),
\end{aligned}
$$

We claim that $a_{n} \geq b_{n}, \ldots, h_{n}$ for $n \geq 1$. By symmetry, it suffices to check that $a_{n+1} \geq b_{n+1}$ for $n \geq 0$. Applying $\frac{1}{2}(x+y) \geq \sqrt{x y}$ to $x=\frac{1}{4}\left(a_{n}+c_{n}+e_{n}+g_{n}\right)$ and $y=\frac{1}{4}\left(b_{n}+d_{n}+f_{n}+h_{n}\right)$ we find that

$$
a_{n+1} \geq \frac{1}{4} \sqrt{\left(a_{n}+c_{n}+e_{n}+g_{n}\right)\left(b_{n}+d_{n}+f_{n}+h_{n}\right)} .
$$

Set $v:=\frac{1}{2}\left(\sqrt{a_{n}}, \sqrt{c_{n}}, \sqrt{e_{n}}, \sqrt{g_{n}}\right)$ and $w:=\frac{1}{2}\left(\sqrt{b_{n}}, \sqrt{d_{n}}, \sqrt{f_{n}}, \sqrt{h_{n}}\right)$. Then

$$
b_{n+1}=v \cdot w \leq\|v\| \cdot\|w\|=\frac{1}{4} \sqrt{\left(a_{n}+c_{n}+e_{n}+g_{n}\right)\left(b_{n}+d_{n}+f_{n}+h_{n}\right)} \leq a_{n+1} .
$$

We find that $a_{n}$ is a decreasing, bounded sequence. Hence $a_{n}$ converges to some number $\alpha$. Similarly, $m_{n}:=\min \left(b_{n}, \ldots, h_{n}\right)$ is an increasing bounded sequence, hence it converges to some number $\mu \leq \alpha$.

Take any $\epsilon>0$. Then $\alpha \leq a_{n}<\alpha+\epsilon$ for all $n \gg 0$. This implies

$$
\alpha \leq a_{n+1}=\frac{1}{8}\left(a_{n}+\cdots+h_{n}\right)<\frac{7}{8}(\alpha+\epsilon)+\frac{1}{8} m_{n},
$$

whence $m_{n}>\alpha-7 \epsilon$ for all $n \gg 0$. Since $\epsilon>0$ is arbitrary, we find that $\mu=\alpha$. This implies that all sequences $a_{n}, \ldots, h_{n}$ converge to $\alpha$.

We continue using the notation $m_{n}:=\min \left(a_{n}, b_{n}, c_{n}, \ldots\right)$.

Theorem 7 Convergence is quadratic in the following sense: $\left|a_{n+1}-m_{n+1}\right| \leq$ $C\left|a_{n}-m_{n}\right|^{2}$ for some constant $C$ not depending on $n$.

Proof We use the idea of the proof of [3, Theorem 8.5]. For notational convenience we take $g=2$, the general case being analogous. We consider the Taylor series of $b_{n+1}=\frac{1}{2}\left(\sqrt{a_{n} b_{n}}+\sqrt{c_{n} d_{n}}\right)$ near $(\ell, \ell, \ell, \ell)$, where $\ell$ is the common limit of the sequences $a_{n}, \ldots, d_{n}$. Note that $\frac{\partial b_{n+1}}{\partial a_{n}}=\cdots=\frac{\partial b_{n+1}}{\partial d_{n}}=\frac{1}{4}$ in $(\ell, \ell, \ell, \ell)$, whereas the 
Hessian equals

$$
H\left(b_{n+1}\right)=\frac{1}{8 \ell}\left(\begin{array}{cccc}
-1 & 1 & 0 & 0 \\
1 & -1 & 0 & 0 \\
0 & 0 & -1 & 1 \\
0 & 0 & 1 & -1
\end{array}\right) .
$$

Using $(x-\ell)^{2}-2(x-\ell)(y-\ell)+(y-\ell)^{2}=(x-y)^{2}$ we compute the Taylor expansion of $b_{n+1}$ around $(\ell, \ldots, \ell)$ to find

$$
\begin{aligned}
b_{n+1}= & \ell+\frac{1}{4}\left(a_{n}-\ell\right)+\cdots+\frac{1}{4}\left(d_{n}-\ell\right)-\frac{1}{16 \ell}\left(\left(a_{n}-b_{n}\right)^{2}+\left(c_{n}-d_{n}\right)^{2}\right) \\
& +O\left(\left|a_{n}-m_{n}\right|^{3}\right) .
\end{aligned}
$$

Hence

$$
a_{n+1}-b_{n+1}=\frac{1}{16 \ell}\left(\left(a_{n}-b_{n}\right)^{2}+\left(c_{n}-d_{n}\right)^{2}\right)+O\left(\left|a_{n}-m_{n}\right|^{3}\right) .
$$

This implies $a_{n+1}-b_{n+1}=O\left(\left|a_{n}-m_{n}\right|^{2}\right)$ and analogously for $a_{n+1}-c_{n+1}$ and $a_{n+1}-d_{n+1}$. The claim follows.

Remark 3 Convergence is also quadratic in the following sense: $\left|a_{n+1}-\ell\right|=$ $O\left(\left|a_{n}-\ell\right|^{2}\right)$ and $\left|m_{n+1}-\ell\right|=O\left(\left|m_{n}-\ell\right|^{2}\right)$, where $\ell$ is the common limit. This follows from the previous theorem, using the estimates $\ell-m_{n} \leq 2^{g}\left(a_{n}-\ell\right)$ and $a_{n}-\ell<2^{g}\left(\ell-m_{n}\right)$ for $n \gg 0$. We leave the details to the reader.

Remark 4 It is conceivable that the convergence of $\ell-b_{n}, \ell-c_{n}, \ldots$ is not quadratic, since by coincidence $\ell-b_{n}$ could be arbitrarily small (even zero) compared to $\ell-$ $b_{n+1}$. It is not clear to us if this can happen for infinitely many $n$. Also, the two extreme sequences $a_{n}$ and $m_{n}$ are monotonous for $n \geq 1$, but it is not clear whether the sequences $b_{n}, c_{n}, \ldots$ are monotonous after finitely many steps.

Open Access This article is distributed under the terms of the Creative Commons Attribution Noncommercial License which permits any noncommercial use, distribution, and reproduction in any medium, provided the original author(s) and source are credited.

\section{Appendix: Maple script}

The integrals above were computed using Maple. The following script computes 500 digits of $\int_{1}^{4} \frac{\mathrm{d} x}{\sqrt{|(x-1)(x-4)(x-9)(x-16)|}}$ in about 2 minutes, whereas the agm yields about 30,000 digits in less than 2 seconds!

Digits:=100: with (student):

$f:=x->1 / \operatorname{sqrt}(\operatorname{abs}((x-1) *(x-4) *(x-9) *(x-16)))$ :

$\mathrm{p}:=\operatorname{Int}(\mathrm{f}(\mathrm{x}), \mathrm{x}=1 \ldots 4$, method=_Gquad) :

$\mathrm{q}:=\operatorname{simplify}\left(\right.$ changevar $\left.\left(\mathrm{x}=1+\mathrm{u}^{\wedge} 2, \mathrm{p}, \mathrm{u}\right)\right)$ :

$r:=\operatorname{simplify}\left(\right.$ changevar $\left.\left(u=\operatorname{sqrt}(3)-v^{\wedge} 2, q, v\right)\right)$ :

evalf $(r)$; 


\section{References}

1. Almkvist, G., Berndt, B.: Gauss, Landen, Ramanujan, the arithmetic-geometric mean, ellipses, $\pi$, and the Ladies Diary. Am. Math. Mon. 95, 581-608 (1988)

2. Borchardt, C.W.: Über das arithmetisch-geometrische Mittel aus vier Elementen. Monatsbericht der königlichen Akademie zu Berlin vom (November 1876)

3. Borwein, J.M., Borwein, P.B.: Pi and the AGM. Wiley-Interscience, New York (1998)

4. Bost, J.-B., Mestre, J.-F.: Moyenne arithmético-géométrique et périodes des courbes de genre 1 et 2, LMENS-88-13 (1988). Département de Mathématiques et d'Informatique, Ecole Normale Supériere

5. Cox, D.A.: The arithmetic-geometric mean of Gauss. Enseign. Math. 30, 275-330 (1984)

6. Cox, D.A.: Gauss and the arithmetic-geometric mean. Not. Am. Math. Soc. 32(2), 147-151 (1985)

7. Donagi, R., Livné, R.: The arithmetic-geometric mean and isogenies for curves of higher genus. Ann. Sc. Norm. Super. Pisa, Cl. Sci., Sr. 4 28(2), 323-339 (1999)

8. Dupont, R.: Moyenne arithmético-géométrique, suites de Borchardt et applictions. Thèse, Ecole Polytechnique (2006). Available at http://www.lix.polytechnique.fr/Labo/Regis.Dupont/

9. Dupont, R.: Moyenne de Borchardt sur les complexes. Preprint

10. Fay, J.D.: Theta Functions on Riemann Surfaces. Lecture Notes in Math., vol. 352. Springer, Berlin (1973)

11. Gauss, C.F.: Determinatio attractionis quam in punctum quodvis positionis datae excerceret planeta si eius massa per totam orbitam ratione temporis quo singulae partes describuntur uniformiter esset dispertita. Gött. Gel. Anz. 3, 331-355 (1818)

12. Griffiths, Ph., Harris, J.: Principles of Algebraic Geometry. Wiley-Interscience, New York (1978)

13. Igusa, J.-I.: Theta Functions. Grundlehren Math. Wiss., vol. 194. Springer, Berlin (1972)

14. Jarvis, F.: Higher genus arithmetic-geometric means. Ramanujan J. 17(1), 1-17 (2008)

15. Lagrange, J.L.: Sur une nouvelle Méthode de Calcul Intégrale pour différentielles affectées d'un radical carré. Mem. Acad. R. Sci. Turin II 2, 252-312 (1784-1785)

16. Lehavi, D., Ritzenthaler, C.: An explicit formula for the arithmetic-geometric mean in genus 3 . Exp. Math. 16(4), 421-440 (2007)

17. Mestre, J.-F.: Moyenne de Borchardt et intégrales elliptiques. C. R. Acad. Sci. Paris, Série I 313, 273-276 (1991)

18. Mumford, D.: Tata Lectures on Theta I. Progress in Mathematics, vol. 28. Birkhäuser, Boston (1983)

19. Mumford, D.: Tata Lectures on Theta II. Progress in Mathematics, vol. 43. Birkhäuser, Boston (1984)

20. Richelot, F.: Essai sur une méthode générale pour déterminer la valeur des intégrales ultra-elliptiques, fondée sur des transformations remarquables de ces transcendentes. C. R. Acad. Sci. Paris 2, 622-627 (1836)

21. Thomae, J.: Beitrag zur Bestimmung von $\theta(0,0, \ldots, 0)$ durch die Klassenmoduln algebraischer Functionen. Crelle J. 71, 201-222 (1870) 